\title{
Electron emission properties of graphene-oxide-semiconductor planar-type electron emission devices
}

Katsuhisa Murakami, Shunsuke Tanaka, Takuya lijima, Masayoshi Nagao, Yoshihiro Nemoto, Masaki Takeguchi, Yoichi Yamada, and Masahiro Sasaki

Citation: Journal of Vacuum Science \& Technology B 36, 02C110 (2018); doi: 10.1116/1.5006866

View online: https://doi.org/10.1116/1.5006866

View Table of Contents: http://avs.scitation.org/toc/jvb/36/2

Published by the American Vacuum Society

\section{Articles you may be interested in}

Graphene-oxide-semiconductor planar-type electron emission device

Applied Physics Letters 108, 083506 (2016); 10.1063/1.4942885

Monolayer graphene-insulator-semiconductor emitter for large-area electron lithography

Applied Physics Letters 110, 233109 (2017); 10.1063/1.4984955

Electron emission from electrically isolated spheres

Journal of Vacuum Science \& Technology B, Nanotechnology and Microelectronics: Materials, Processing, Measurement, and Phenomena 36, $02 \mathrm{C} 102$ (2018); 10.1116/1.5004748

Local current-voltage estimation and characteristization based on field emission image processing of large-area field emitters

Journal of Vacuum Science \& Technology B, Nanotechnology and Microelectronics: Materials, Processing, Measurement, and Phenomena 36, $02 \mathrm{C} 106$ (2018); 10.1116/1.5007006

Interface-induced perpendicular magnetic anisotropy of Co nanoparticles on single-layer h-BN/Pt(111)

Applied Physics Letters 112, 022407 (2018); 10.1063/1.5010836

Characterization of thin carbon films capable of low-field electron emission

Journal of Vacuum Science \& Technology B, Nanotechnology and Microelectronics: Materials, Processing, Measurement, and Phenomena 36, $02 \mathrm{C} 108$ (2018); 10.1116/1.5009906

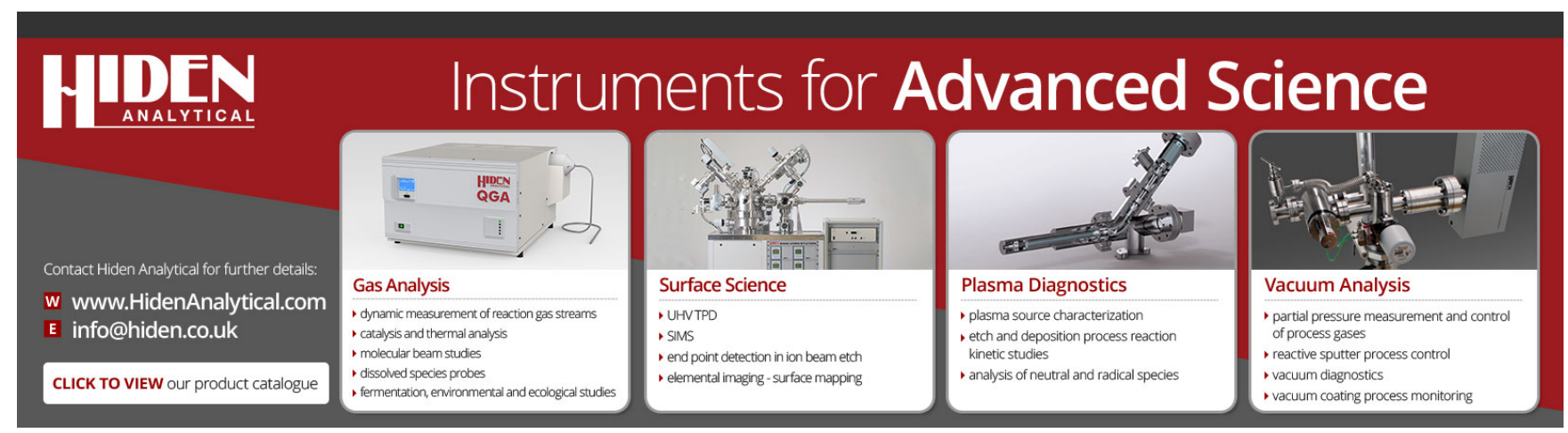




\title{
Electron emission properties of graphene-oxide-semiconductor planar-type electron emission devices
}

\author{
Katsuhisa Murakamia) \\ National Institute of Advanced Industrial Science and Technology, 1-1-1 Umezono, Tsukuba, Ibaraki \\ 305-8568, Japan; Institute of Applied Physics, Graduate School of Pure and Applied Sciences, University \\ of Tsukuba, 1-1-1 Tennodai, Tsukuba, Ibaraki 305-8573, Japan; and National Institute for Materials Science, \\ 1-2-1 Sengen, Tsukuba, Ibaraki 305-0047, Japan \\ Shunsuke Tanaka and Takuya lijima \\ Institute of Applied Physics, Graduate School of Pure and Applied Sciences, University of Tsukuba, \\ 1-1-1 Tennodai, Tsukuba, Ibaraki 305-8573, Japan \\ Masayoshi Nagao \\ National Institute of Advanced Industrial Science and Technology, 1-1-1 Umezono, Tsukuba, \\ Ibaraki 305-8568, Japan \\ Yoshihiro Nemoto and Masaki Takeguchi \\ National Institute for Materials Science, 1-2-1 Sengen, Tsukuba, Ibaraki 305-0047, Japan \\ Yoichi Yamada and Masahiro Sasaki \\ Institute of Applied Physics, Graduate School of Pure and Applied Sciences, University of Tsukuba, \\ 1-1-1 Tennodai, Tsukuba, Ibaraki 305-8573, Japan
}

(Received 28 September 2017; accepted 8 February 2018; published 27 February 2018)

\begin{abstract}
The electron emission properties of planar-type electron emission devices based on a grapheneoxide-semiconductor (GOS) structure before and after vacuum annealing were investigated. The fluctuation of the electron emission current was around $0.07 \%$, which is excellent stability compared to the conventional field emitter array. The GOS devices were operable in very low vacuum of $10 \mathrm{~Pa}$ without any deterioration of their electron emission properties. Improvement of the electron emission properties of the GOS devices was achieved by vacuum annealing at $300^{\circ} \mathrm{C}$. The electron emission efficiency of the GOS type electron emission devices reached $2.7 \%$ from $0.2 \%$ after vacuum annealing. The work function of the graphene electrode was found to decrease $0.26 \mathrm{eV}$ after vacuum annealing by Kelvin force probe microscopy analysis. These results indicated that the improvement of the electron emission efficiency of the GOS devices by vacuum annealing is due to the decrease in the work function of the graphene electrode. Published by the AVS. https://doi.org/10.1116/1.5006866
\end{abstract}

\section{INTRODUCTION}

A planar type electron emission device based on a metal-oxide-semiconductor (MOS) structure $^{1}$ has great potential for applications utilizing electron beams such as field emission displays, ${ }^{2}$ high-sensitive image sensors, ${ }^{3}$ field emission lamps, ${ }^{4,5}$ and flat-panel x-ray sources ${ }^{6,7}$ since it can be operated in low vacuum and low voltage conditions ${ }^{1}$ compared to a conventional field emitter array. ${ }^{8}$ However, its electron emission efficiency, which is defined as the ratio of emission current from the topmost gate electrode into vacuum divided by the total current flow through the semiconductor substrate, is typically very low of around $0.002 \% .^{9}$ There are three primary factors for the low electron emission efficiency of the MOS type electron emission devices, as shown in the band diagram of the MOS type devices during electron emission (Fig. 1). The first factor is electron inelastic scattering within the oxide layer. When a gate bias voltage is applied to the metal electrode, the potential barrier of the oxide layer becomes a triangle shape, and by increasing the voltage, the barrier width for an electron tunneling decreases. Then, electrons on the

${ }^{a)}$ Electronic mail: murakami.k@aist.go.jp conduction band of the $\mathrm{Si}$ substrate tunnel into the oxide layer. While the tunneled electrons travel through the oxide layer under the influence of the electric field, electrons are scattered and lose their energy. ${ }^{9}$ The second factor is electron scattering within the metal gate electrode. ${ }^{9}$ The third factor is the electrons which have lower energy than the work function of the metal gate electrode collected by the gate electrode as the gate current of the MOS devices. ${ }^{10}$ To improve the electron emission efficiency of MOS devices, suppression of electron scattering within the oxide and the electrode is very important. Another strategy is to use low work function materials as a gate electrode. In our previous study, a high emission current density of $1-100 \mathrm{~mA} / \mathrm{cm}^{2}$ was achieved by suppressing the inelastic electron scattering within the topmost gate electrode using a graphene gate electrode, while maintaining a relatively high electron emission efficiency of $0.1 \%-1 \%$, which is 2 orders of magnitude higher than the conventional MOS planar-type electron emission devices. ${ }^{11}$ In this study, detailed electron emission properties of the graphene-oxide-semiconductor (GOS) type planar electron emission device (e.g., operating vacuum pressure dependence and stability of electron emission current) and further improvement of its electron emission properties by vacuum annealing are discussed. 


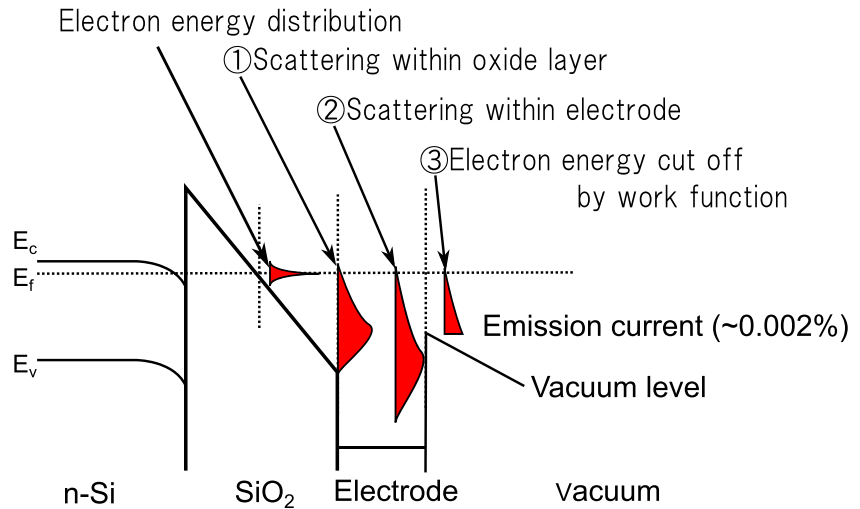

FIG. 1. (Color online) Energy band diagram of the conventional MOS type electron emission device during electron emission.

\section{EXPERIMENT}

A highly doped n-type Si substrate with a thermal oxide thickness of $300 \mathrm{~nm}$ was used. The electron emission area of $10-100 \mu \mathrm{m}$ square was fabricated using conventional photolithography and wet etching. The thin oxide layer with a thickness of $8 \mathrm{~nm}$ was then grown by thermal oxidation at $900^{\circ} \mathrm{C}$ after conventional RCA cleaning. A graphene electrode with a thickness of $7 \mathrm{~nm}$ was synthesized on the entire surface of the substrate by gallium vapor-assisted chemical vapor deposition $(\mathrm{CVD})$ at $1050^{\circ} \mathrm{C}^{12}$ The $\mathrm{Au} / \mathrm{Cr}$ contact electrode was then fabricated using conventional photolithography, radio frequency sputtering, and the lift-off process. Finally, the graphene electrode was partially etched by photolithography and $\mathrm{O}_{2}$ plasma for device isolation. Their detailed fabrication processes were described elsewhere. ${ }^{11}$

The electron emission characteristics were measured in a vacuum chamber at the pressures of $10,10^{-4}$, and $10^{-6} \mathrm{~Pa}$ evacuated by a turbomolecular pump and/or a scroll pump, respectively. An anode electrode of a metal plate for applying a voltage of $1 \mathrm{kV}$ was placed $5 \mathrm{~mm}$ away from the gate electrode. In the case of the operating vacuum pressure dependence measurement, the anode bias was $100 \mathrm{~V}$ in order to prevent electric discharge at low vacuum. The cycle of evacuation and vent of the measurement chamber and electron emission measurement at each vacuum level were performed twice. In addition, the electron emission properties at low vacuum conditions of $10 \mathrm{~Pa}$ were measured three times. The GOS devices were annealed at $200-300^{\circ} \mathrm{C}$ in the loadlock chamber at a base pressure of $\sim 10^{-4} \mathrm{~Pa}$.

The work function of the graphene electrode before and after annealing was measured by Kelvin force probe microscopy with a Pt/Ir coated Si probe in the atmosphere using the PeakForce KPFM mode of Bruker Dimension ICON.

\section{RESULTS AND DISCUSSION}

Figure 2 shows the scanning electron microscopy (SEM) image of the typical GOS emission devices. The uniform dark contrast region shows the graphene electrode. The graphene electrode directly fabricated on the $\mathrm{SiO}_{2}$ surface by Ga-vapor assisted CVD was found to uniformly cover the surface of the devices without any cracks and wrinkles,

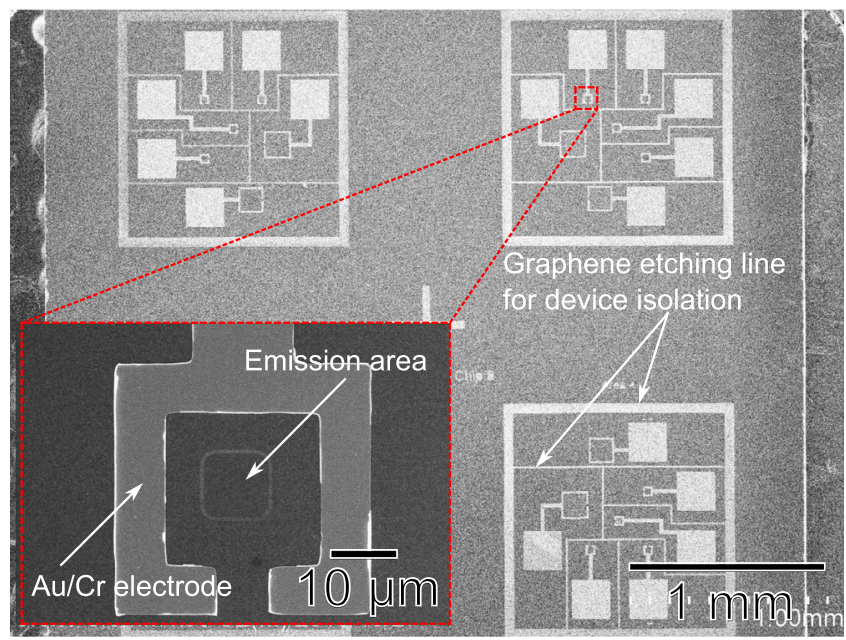

FIG. 2. (Color online) SEM image of the GOS devices. The inset shows the enlarged view of the electron emission area.

which is the inherent issue for the conventional graphene transfer processes of the CVD graphene on a $\mathrm{Cu}$ substrate using a resist polymer and $\mathrm{Cu}$ etching solutions. ${ }^{13} \mathrm{In}$ addition, the direct CVD method of graphene on an insulator substrate would allow us to integrate the GOS devices in a wafer scale, which is an advantage for future industrial applications, although the limitation of the substrate size for our CVD equipment is presently $20 \mathrm{~mm}$ square. The CVD system of graphene direct synthesis on an insulator substrate for a 4-in. wafer is now under development in our group.

Figure 3 shows the typical current-voltage curve, the Fowler-Nordheim (FN) plots, and electron emission stability of the GOS devices with an emission area of $100 \mu \mathrm{m}$ square. Emission current was detected at the gate bias of around $9 \mathrm{~V}$ and reached $10 \mathrm{~mA} / \mathrm{cm}^{2}$ at $20 \mathrm{~V}$. The emission efficiency reached the maximum (approximately $0.44 \%$ ) at $13 \mathrm{~V}$ and then slowly decreased with the gate voltage. Although only the data obtained from the GOS device with an emission area of $100 \mu \mathrm{m}$ square were shown here, it was confirmed that the current scales linearly increase with the emission area from 10 to $100 \mu \mathrm{m}$ square, which means that electrons emitted from whole of the emission area. The FN plot of anode and cathode current showed a linear dependence, which indicates that measured emission current is originated from FN tunneling. The emission current fluctuation was approximately $0.07 \%$ and showed no spike noise at a gate bias voltage of $15 \mathrm{~V}$ under direct current operation. The integration time of the source measure unit for stability measurements was 1 power line cycles $(50 \mathrm{~Hz})$. The root mean square noise of the current measurement is $\pm 0.3 \mathrm{pA}$, which corresponds to $\pm 3 \mathrm{nA} / \mathrm{cm}^{2}$ calculated from the electron emission area of $100 \mu \mathrm{m}$ square. Therefore, measurement error of the source measure unit hardly affects the stability measurement at the current density level (i.e., around $70 \mu \mathrm{A} /$ $\mathrm{cm}^{2}$ ) of this experiment. The reason for the excellent stability of electron emission current is that the potential barrier to the electron tunneling is at the interface between the semiconductor substrate and the oxide layer and is not exposed to a vacuum unlike the conventional field emitter devices. 

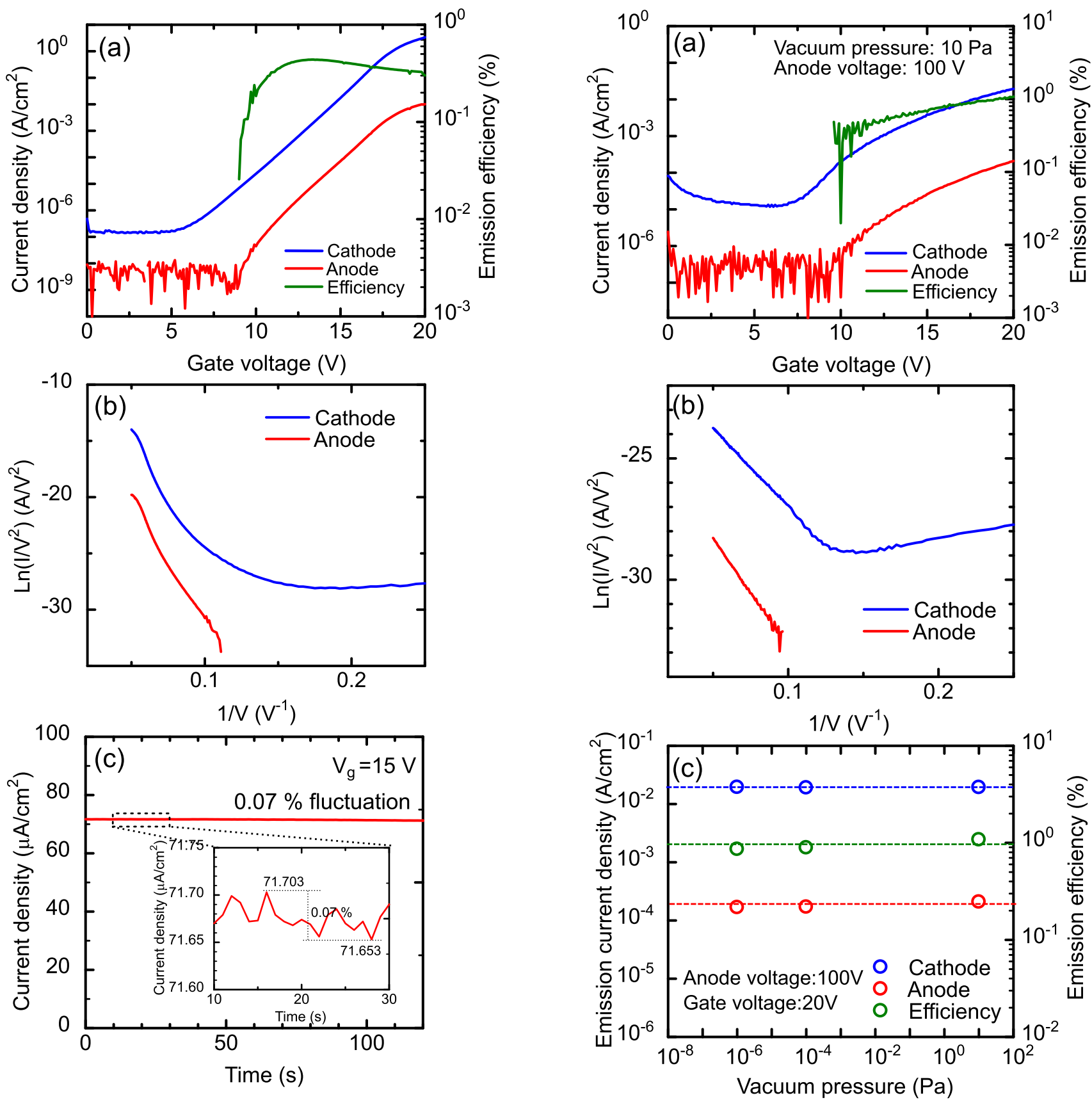

FIG. 3. (Color online) (a) Typical current-voltage curve, (b) FN plots, and (c) electron emission stability of the GOS device with an emission area of $100 \mu \mathrm{m}^{2}$.

Therefore, the fluctuation of the potential barrier by adsorbed molecules, which is the main reason for unstable electron emission of the conventional field emitters, is avoided. Another possible reason is the large electron emission area of the GOS devices compared to the conventional field emitters. In the case of the conventional field emitter, the stability of the emission current improves by increasing the number of integrated field emitters. ${ }^{14}$ The large electron emission area would be regarded as an array of emission sites like a field emitter array.

Figure 4 shows the typical current-voltage curve of the GOS device operated at $10 \mathrm{~Pa}$, the FN plots, and the anode and cathode current of the GOS device at the gate bias

FIG. 4. (Color online) (a) Typical current-voltage curve of the GOS device operated at $10 \mathrm{~Pa}$, (b) FN plots, and (c) Anode current, cathode current, and electron emission efficiency of the GOS device at the gate bias voltage of $20 \mathrm{~V}$ as a function of a vacuum pressure.

voltage of $20 \mathrm{~V}$ as a function of a vacuum pressure. The electron emission properties of the GOS devices did not deteriorate even at a vacuum pressure of $10 \mathrm{~Pa}$. As described before, the potential barrier for the electron tunneling of the GOS devices is internal to the device structure. Therefore, high electric fields for electron tunneling can be applied at even atmospheric pressure without an electric discharge. Consequently, the GOS devices can be operated at a high pressure of $10 \mathrm{~Pa}$. In fact, the planar type electron emission devices based on a MOS structure can be operated in atmospheric pressure ${ }^{15}$ and liquid. ${ }^{16}$ This unique feature can 
provide novel applications utilizing electron beams, such as the hydrogen generation by hot electron irradiation to water. ${ }^{16}$

Figure 5 shows the typical current-voltage curve of the GOS devices, the FN plots, and the data for electron emission efficiency of the GOS devices before and after vacuum annealing. The gate voltage, at which the anode current was detected in our measurement set-up, shifted to $8 \mathrm{~V}$ from $10 \mathrm{~V}$ after $300{ }^{\circ} \mathrm{C}$ annealing for $1 \mathrm{~h}$. The anode current density at the gate voltage of $13 \mathrm{~V}$ increased from $100 \mu \mathrm{A} / \mathrm{cm}^{2}$ to $1.4 \mathrm{~mA} / \mathrm{cm}^{2}$ after $300^{\circ} \mathrm{C}$ annealing, while the cathode current density was almost unchanged. Consequently, the electron emission efficiency improved ten times due to $300^{\circ} \mathrm{C}$
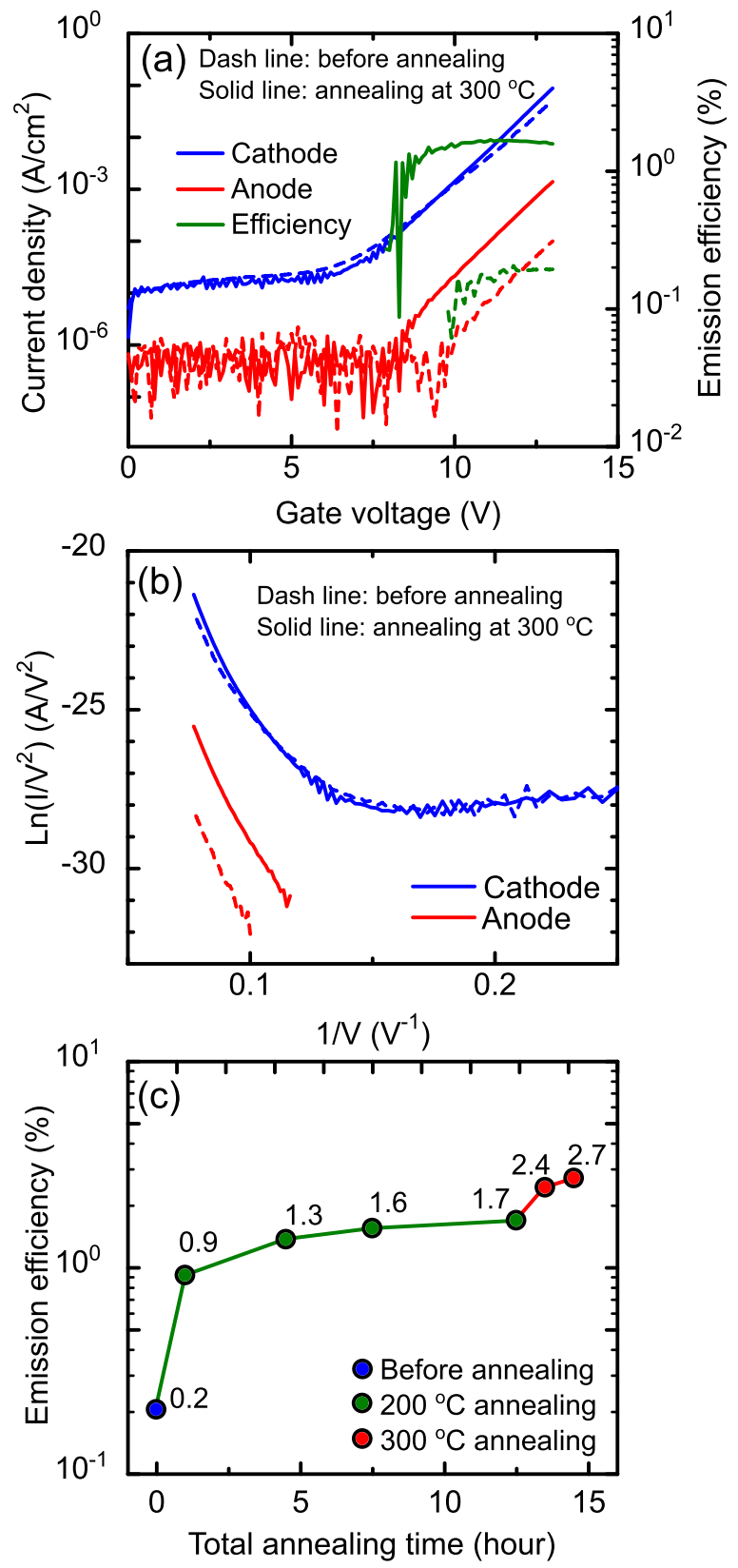

FIG. 5. (Color online) (a) Typical current-voltage curve and (b) FN plots of the GOS devices before and after annealing at $300^{\circ} \mathrm{C}$ for $1 \mathrm{~h}$. (c) Series data of electron emission efficiency of the GOS device before and after annealing. annealing. The efficiency after annealing at $200^{\circ} \mathrm{C}$ was found to increase with annealing time. The efficiency reached $1.7 \%$ from $0.2 \%$ by annealing at $200^{\circ} \mathrm{C}$ for $12.5 \mathrm{~h}$. The additional annealing at $300^{\circ} \mathrm{C}$ further improved the electron emission efficiency and reached $2.7 \%$. The emission efficiency was slightly deteriorated by air exposure after vacuum annealing. However, electron emission efficiency retained a higher value than the initial state even after exposing the GOS devices to air for 5 days. Such a long air exposure time is enough to reach the same surface coverage of gas molecules as the initial conditions. These results indicate that the origin of the improvement of the efficiency of the GOS devices is not only the desorption of the adsorbed molecules, such as water and oxygen, on the surface of the graphene gate electrode.

Figure 6 shows the topography image of the GOS device and the line profiles of the work function difference of the GOS devices with respect to the Pt/Ir coated Si probe before and after annealing. The values of the work function of the GOS device were estimated using the work function of Pt of $5.65 \mathrm{eV} .{ }^{17}$ The estimated values of the work function of the graphene electrode and the Au contact electrode before and after annealing are summarized in Table I.

The work function of the graphene gate electrode was found to decrease by $0.26 \mathrm{eV}$ after vacuum annealing, which
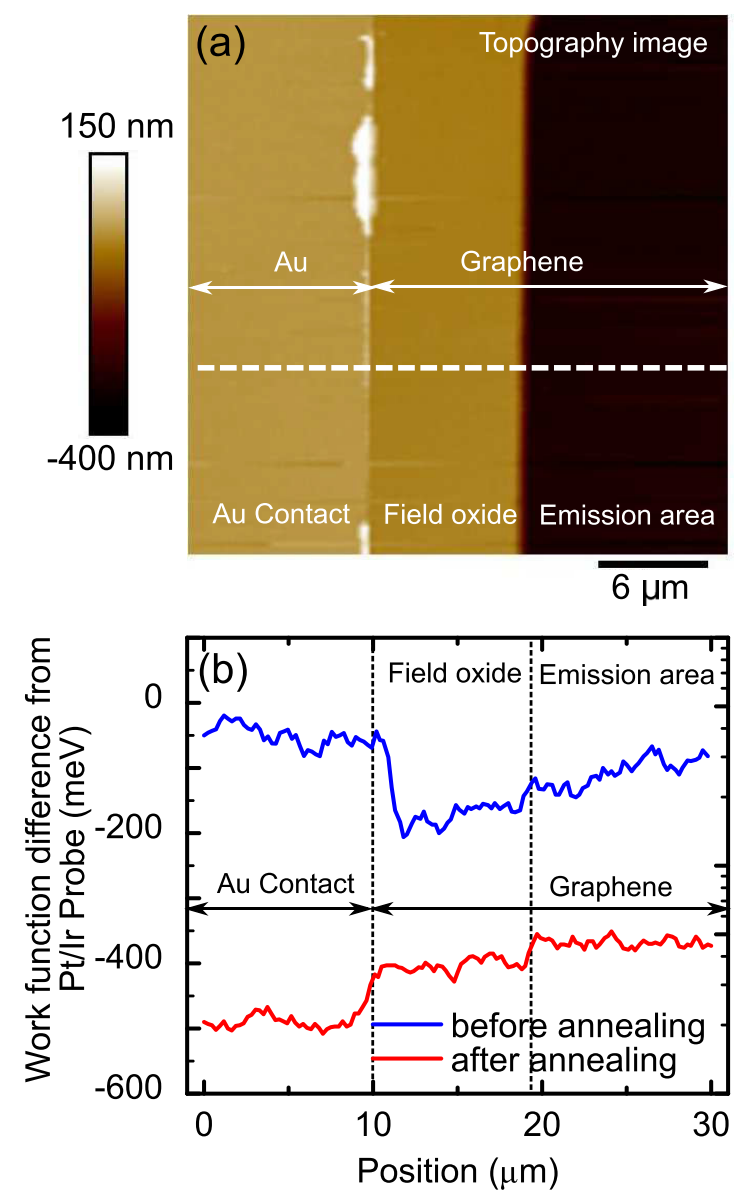

FIG. 6. (Color online) (a) Topography image of the GOS device; (b) Line profiles of the work function difference of the GOS devices with respect to the Pt/Ir coated Si probe before and after annealing. 
TABLE I. Work function of the graphene electrode of the emission area and the Au contact electrode of the GOS device before and after annealing.

\begin{tabular}{lcc}
\hline \hline & \multicolumn{2}{c}{ Work function (eV) } \\
\cline { 2 - 3 } & Graphene (Emission area) & Au contact \\
\hline Before annealing & 5.54 & 5.6 \\
After annealing & 5.28 & 5.16 \\
\hline \hline
\end{tabular}

is the conceivably main factor for the improvement of the electron emission efficiency of the GOS device. The work function of the Au contact electrode was also decreased $0.44 \mathrm{eV}$ after vacuum annealing. In addition, the work function of the Au contact electrode after vacuum annealing was $5.16 \mathrm{eV}$, which is consistent with the work function of $\mathrm{Au}$ reported in the literature. ${ }^{17}$ Therefore, the reduction of the work function by vacuum annealing observed in our experiment was not a peculiar phenomenon on graphene. The possible reason for the reduction of the work function of the GOS device by vacuum annealing is the removal of the contaminations on the surface of the devices, such as residual photo resist polymers used in the device fabrication processes. However, the work function of the graphene electrode after vacuum annealing is $5.28 \mathrm{eV}$, which is still higher than its theoretical value of $4.5-4.6 \mathrm{eV} \cdot{ }^{18,19}$ One possible explanation for the higher work function of the graphene gate electrode is that the hole doping occurred from the adsorbed oxygen on the graphene surface. ${ }^{20}$ Another possible reason is that the graphene synthesized by Ga-vapor assisted CVD shows p-type characteristics. ${ }^{12}$ These lead to the higher effective work function of the graphene gate electrode. Therefore, n-type doping of the graphene gate electrode by a chemical surface treatment would further improve the electron emission efficiency of the GOS devices. ${ }^{21-23}$

\section{SUMMARY AND CONCLUSIONS}

The electron emission properties of the GOS devices before and after vacuum annealing were investigated. The fluctuation of the electron emission current is around $0.07 \%$, which is excellent stability compared to the conventional field emitter array. In addition, the GOS devices were operable in very low vacuum of $10 \mathrm{~Pa}$ without any deterioration of their electron emission properties. The electron emission efficiency was found to be improved by vacuum annealing at $200-300{ }^{\circ} \mathrm{C}$. The maximum electron emission efficiency was $2.7 \%$ after vacuum annealing at $300^{\circ} \mathrm{C}$. The work function of the surface of the graphene gate electrode was found to decrease, which is perhaps the main contribution to the improvement of the electron emission efficiency of the GOS devices. The planar type electron emission devices with high electron emission efficiency operable in low voltage and low vacuum conditions would provide novel and widespread applications utilizing electron beams.

\section{ACKNOWLEDGMENTS}

This work was partly supported by the Ministry of Education, Culture, Sports, Science and Technology (MEXT) of Japan through a Grant-in-Aid for Young Scientists (A) (Grant No. 15H05522), a Grant-in-Aid for challenging Exploratory Research (Grant No.16K14223), a Grant-in-Aid for Scientific Research (A) (Grant No.15H02195), and by Nanotech Career-up Alliance (Nanotech CUPAL). Support was also received from the NIMS Molecule and Material Synthesis Platform, the NIMS Microstructural Characterization Platform, and the University of Tsukuba Nanofabrication Platform in the Nanotechnology Platform Project sponsored by MEXT, Japan.

${ }^{1}$ K. Yokoo, H. Tanaka, S. Sato, J. Murota, and S. Ono, J. Vac. Sci. Technol., B 11, 429 (1993).

${ }^{2}$ T. Kusunoki, M. Suzuki, M. Sagawa, Y. Mikami, E. Nishimura, M. Ikeda, T. Hirano, and K. Tsuji, J. Vac. Sci. Technol., B 30, 041202 (2012).

${ }^{3}$ N. Negishi et al., J. Vac. Sci. Technol., B 26, 711 (2008).

${ }^{4}$ Y. Nakajima, T. Uchida, H. Toyama, A. Kojima, B. Gelloz, and N. Koshida, Jpn. J. Appl. Phys., Part 1 43, 2076 (2004).

${ }^{5}$ T. Ichihara, T. Hatai, and N. Koshida, J. Vac. Sci. Technol., B 27, 772 (2009).

${ }^{6}$ C. M. Posada, E. J. Grant, R. Divan, A. V. Sumant, D. Rosenmann, L. Stan, H. K. Lee, and C. H. Castano, J. Appl. Phys. 115, 134506 (2014).

${ }^{7}$ D. Chen, X. Song, Z. Zhang, Z. Li, J. She, S. Deng, N. Xu, and J. Chen, Appl. Phys. Lett. 107, 243105 (2015).

${ }^{8}$ K. Murakami and M. Takai, Microelectron. Eng. 132, 74 (2015).

${ }^{9}$ K. Yokoo, S. Sato, G. Koshita, I. Amano, J. Murota, and S. Ono, J. Vac. Sci. Technol., B 12, 801 (1994).

${ }^{10}$ H. Mimura, Y. Neo, H. Shimawaki, Y. Abe, K. Tahara, and K. Yokoo, Appl. Phys. Lett. 88, 123514 (2006).

${ }^{11}$ K. Murakami, S. Tanaka, A. Miyashita, M. Nagao, Y. Nemoto, M. Takeguchi, and J. Fujita, Appl. Phys. Lett. 108, 83506 (2016).

${ }^{12}$ K. Murakami, S. Tanaka, A. Hirukawa, T. Hiyama, T. Kuwajima, E. Kano, M. Takeguchi, and J. Fujita, Appl. Phys. Lett. 106, 093112 (2015).

${ }^{13}$ A. Reina, H. Son, L. Jiao, B. Fan, M. Dresselhaus, Z. Liu, and J. Kong, J. Phys. Chem. C 112, 17741 (2008).

${ }^{14}$ J. Itoh, Appl. Surf. Sci. 111, 194 (1997).

${ }^{15}$ T. Ohta, A. Kojima, H. Hirakawa, T. Iwamatsu, and N. Koshida, J. Vac. Sci. Technol., B 23, 2336 (2005).

${ }^{16}$ N. Koshida, T. Ohta, and B. Gelloz, Appl. Phys. Lett. 90, 163505 (2007).

${ }^{17}$ D. E. Eastman, Phys. Rev. B: Condens. Matter 2, 1 (1970).

${ }^{18}$ N. Ooi, A. Rairkar, and J. Adams, Carbon 44, 231 (2006).

${ }^{19}$ S. Sque, R. Jones, and P. Briddon, Phys. Status Solidi 204, 3078 (2007).

${ }^{20}$ Y. Sato, K. Takai, and T. Enoki, Nano Lett. 11, 3468 (2011).

${ }^{21}$ T. Kusunoki and M. Suzuki, Appl. Surf. Sci. 100/101, 207 (1996).

${ }^{22}$ J. Chang, W. Lin, J. Taur, T. Chen, G. Liao, T. Pi, M. Chen, and C. Wu, ACS Appl. Mater. Interfaces 7, 17155 (2015).

${ }^{23}$ H. Yuan et al., Nano Lett. 15, 6475 (2015). 